\title{
A Practical Method for the Synthesis of Indolylaryl- and Bisindolylmaleimides
}

Sudipta Roy, Sujata Roy and Gordon W. Gribble*

\author{
Department of Chemistry, Dartmouth College, \\ Hanover, New Hampshire 03755. \\ ggribble@dartmouth.edu
}

Supplemental Information

Table of Contents:

Experimental Procedure:

S2-S7

Spectra for Compounds:

S8-S21 


\section{Experimental}

\section{General:}

Melting points were determined with a Mel-Temp Laboratory Device apparatus and are uncorrected. IR spectra were recorded on a Perkin-Elmer 600 series FTIR spectrophotometer. ${ }^{1} \mathrm{H}$-and ${ }^{13} \mathrm{C}$-NMR spectra were recorded on a Varian XL-500 Fourier transform NMR spectrometer. Both low- and high resolution mass spectra were carried out at the Mass Spectrometry Laboratory, School of Chemical Sciences, University of Illinois at Urbana Champaign. Anhydrous THF was prepared by a solvent purification system. All other solvents (analytical grade) including anhydrous solvents and reagents were used as received. All methyl arylacetates, except $N$-methylindole-3-acetate, are purchased from Aldrich, Acros or Alfa-Aesar. All experiments were performed under a nitrogen atmosphere.

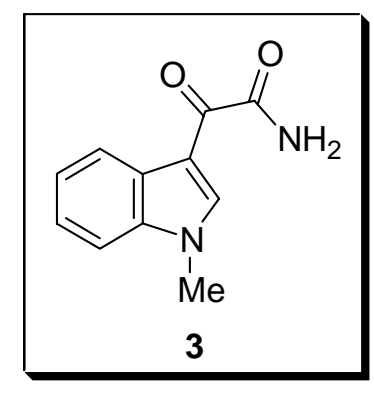

$N$-Methylindole-3-glyoxylamide (3): To a stirred solution of $N$-methylindole ${ }^{1}$ (3.32 g, $25.3 \mathrm{mmol})$ in anhydrous ether $(200 \mathrm{~mL})$ at $0{ }^{\circ} \mathrm{C}$ was added dropwise a solution of oxalyl chloride (4.4 mL,50.6 mmol). The reaction mixture was stirred for $30 \mathrm{~min}$ at the same temperature. The orange solid was filtered-off, washed with cold ether and air-dried. The crude solid product was used in the next step without any further purification. The crude 1-methylindole-3-glyoxylyl chloride was added in small portions to a stirred concentrated ammonium hydroxide solution $(70 \mathrm{~mL})$ at $0{ }^{\circ} \mathrm{C}$. The mixture was stirred for $30 \mathrm{~min}$ at the same temperature. The white precipitate was collected by filtration, washed with water and recrystallized first from methanol and then from dichloromethane to yield the desired product (3.83 g, 75\%) as a white solid: mp 186-188 ${ }^{\circ} \mathrm{C}$ (lit ${ }^{2} 185-187{ }^{\circ} \mathrm{C}$ ); ${ }^{1} \mathrm{H}-\mathrm{NMR}$ (DMSO-d ${ }_{6}$ ): $\delta 8.76$ (s, 1H), 8.25-8.27 (m, 1H), 8.12 (brs, 1H), 7.76 (brs, 1H), 7.56-7.58 (m, 1H), 7.28-7.34 (m, 2H), 3.90 (s, 3H); ${ }^{13} \mathrm{C}-\mathrm{NMR}$ (DMSO-d 6 ): $\delta 182.4,166.0,141.7$, 137.0, 126.7, 123.5, 122.9, 121.4, 111.0, 110.9, 33.4. 


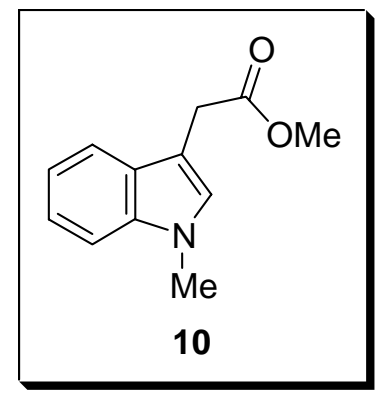

$N$-Methylindole-3-acetate (10): A solution of $N$-methylindole-3-acetic acid ${ }^{3}$ (1.89 g, 10 mmol) in methanol (60 mL) and concentrated $\mathrm{H}_{2} \mathrm{SO}_{4}(3 \mathrm{~g})$ was stirred at rt for $1.5 \mathrm{~h}$. It was then poured into ice-cold saturated aqueous $\mathrm{NaHCO}_{3}(225 \mathrm{~mL})$ with vigorous stirring. It was extracted with chloroform ( 2 x $100 \mathrm{~mL})$. The combined organic phase was washed with water $(100 \mathrm{~mL})$, brine $(100 \mathrm{~mL})$ and dried $\left(\mathrm{Na}_{2} \mathrm{SO}_{4}\right)$. The solvent was evaporated and the residue was purified by column chromatography on silica-gel (3:1 hexanes: ethyl acetate) to yield the desired product ${ }^{4}(1.91 \mathrm{~g}, 94 \%)$ as a yellowish oil: IR (thin film): 3052, 2949, 1731, 1616, 1553, 1473, 1434, 1376, 1332, 1164, 1063, 1012, $741 \mathrm{~cm}^{-1}$; ${ }^{1} \mathrm{H}-\mathrm{NMR}\left(\mathrm{CDCl}_{3}\right): \delta$ 7.68-7.70 (m, 1H), 7.31-7.37 (m, 2H), 7.20-7.24 (m, 1H), 7.09 (s, 1H), 3.85 (s, 2H), 3.79 (s, 3H), 3.77 (s, 3H); ${ }^{13} \mathrm{C}-\mathrm{NMR}\left(\mathrm{CDCl}_{3}\right): \delta 172.9,137.2$, 128.1, 128.0, 122.1, 119.5, 119.3, 109.6, 107.0, 52.3, 32.9, 31.4.

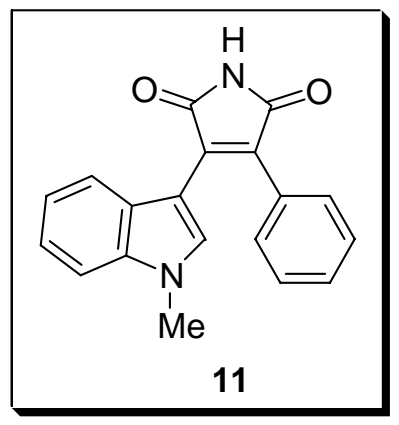

3-(1-Methyl-1H-indol-3-yl)-4-phenyl-1H-pyrrole-2,5-dione (11): To a mixture of $\mathrm{N}$ methylindole-3-glyoxylamide $(0.21 \mathrm{~g}, 1 \mathrm{mmol})$ and methyl phenylacetate $(0.4 \mathrm{~mL}, 2.7$ $\mathrm{mmol})$ in THF $(8 \mathrm{~mL})$ at $0{ }^{\circ} \mathrm{C}$ was slowly added potassium tert-butoxide $(4 \mathrm{~mL}, 1 \mathrm{M}$ in THF, $4 \mathrm{mmol}$ ). The mixture was stirred for $15 \mathrm{~h}$ allowing the mixture to warm slowly to rt. It was poured into brine-water $(100 \mathrm{~mL}, 1: 1)$ and extracted with ethyl acetate $(3 \times 50$ $\mathrm{mL})$. The organic phase was washed with brine $(50 \mathrm{~mL})$ and dried $\left(\mathrm{Na}_{2} \mathrm{SO}_{4}\right)$. The solvent was evaporated and the residue was recrystallized from ethyl acetate to yield the desired product 11 (0.26 g, 86\%) as an orange solid: mp 256-257 ${ }^{\circ} \mathrm{C}$; IR (thin film): 1756, 1697,

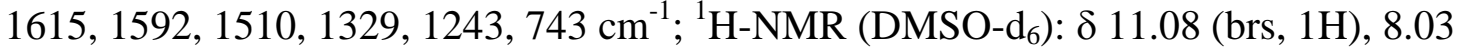
(s, 1H), 7.47 (d, 1H, $J=7.9$ Hz), 7.38-7.39 (m, 2H), 7.30-7.35 (m, 3H), 7.11 (t, 1H, $J=$ $7.6 \mathrm{~Hz}), 6.70$ (t, 1H, $J=7.7 \mathrm{~Hz}), 6.27$ (d, $1 \mathrm{H}, J=7.9 \mathrm{~Hz}), 3.90(\mathrm{~s}, 3 \mathrm{H}) ;{ }^{13} \mathrm{C}-\mathrm{NMR}$ (DMSO-d $\mathrm{d}_{6}$ ): $\delta$ 172.5, 172.3, 137.1, 134.8, 132.0, 130.6, 129.6, 128.6, 128.5, 128.1, 124.3, 122.1, 121.3, 120.0, 110.6, 103.1, 33.0. LRMS (EI): m/z 302 (M , 100\%), 231, 149, 115; HRMS (EI): calcd for $\mathrm{C}_{19} \mathrm{H}_{14} \mathrm{~N}_{2} \mathrm{O}_{2}$ : 302.1055, found: 302.1058 . 


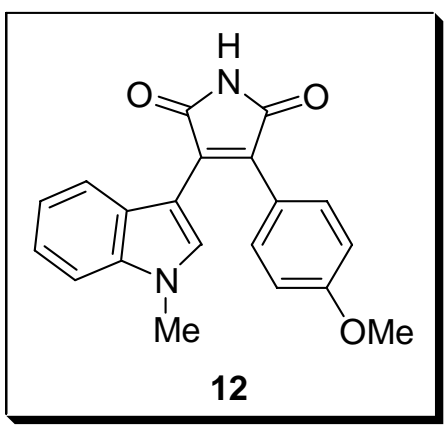

3-(4-Methoxyphenyl)-4-(1-methyl-1H-indol-3-yl)-1H-pyrrole-2,5-dione (12): To a mixture of $\mathrm{N}$-methylindole-3-glyoxylamide $(0.21 \mathrm{~g}, 1 \mathrm{mmol})$ and methyl 4methoxyphenylacetate $(0.49 \mathrm{~g}, 2.7 \mathrm{mmol})$ in THF $(8 \mathrm{~mL})$ at $0{ }^{\circ} \mathrm{C}$ was slowly added potassium tert-butoxide ( $4 \mathrm{~mL}, 1 \mathrm{M}$ in THF, $4 \mathrm{mmol}$ ). The mixture was stirred for $14 \mathrm{~h}$ allowing the mixture to warm slowly to rt. It was poured into brine-water (100 mL, 1:1) and extracted with ethyl acetate $(3 \times 50 \mathrm{~mL})$. The organic phase was washed with brine $(50 \mathrm{~mL})$ and dried $\left(\mathrm{Na}_{2} \mathrm{SO}_{4}\right)$. The solvent was evaporated and the residue was recrystallized from ethyl acetate to yield the desired product $12(0.27 \mathrm{~g}, 81 \%)$ as a dark orange solid: mp $265-267{ }^{\circ} \mathrm{C}$; IR (thin film): 1700, 1600, 1506, 1328, 1244, $739 \mathrm{~cm}^{-1}$; ${ }^{1} \mathrm{H}-$ NMR (DMSO-d $)_{6}$ ): 11.01 (s, 1H), 7.97 (s, 1H), 7.47 (d, 1H, $J=8.2 \mathrm{~Hz}$ ), 7.37 (d, 2H, $J=$ $7.8 \mathrm{~Hz}$ ), 7.12 (t, 1H, $J=7.4 \mathrm{~Hz}), 6.87$ (d, 2H, $J=8.9 \mathrm{~Hz}), 6.76$ (t, 1H, $J=7.6 \mathrm{~Hz}), 6.42$ (d, $1 \mathrm{H}, J=8.2 \mathrm{~Hz}$ ), 3.89 (s, 3H), 3.74 (s, 3H); ${ }^{13} \mathrm{C}-\mathrm{NMR}\left(\mathrm{DMSO}_{\mathrm{d}}\right.$ ): $\delta 172.8,172.4$, 159.5, 137.0, 134.3, 131.1, 130.4, 128.8, 124.4, 122.8, 122.0, 121.4, 120.0, 113.6, 110.5, 103.2, 55.2, 33.0; LRMS (ESI+): $\mathrm{m} / \mathrm{z} 355$ [M+Na] ${ }^{+}, 333$ [M+H] ${ }^{+}$; HRMS (ESI+): calcd for $\mathrm{C}_{20} \mathrm{H}_{17} \mathrm{~N}_{2} \mathrm{O}_{3}$ : 333.1239 [M+H] found: 333.1249 .

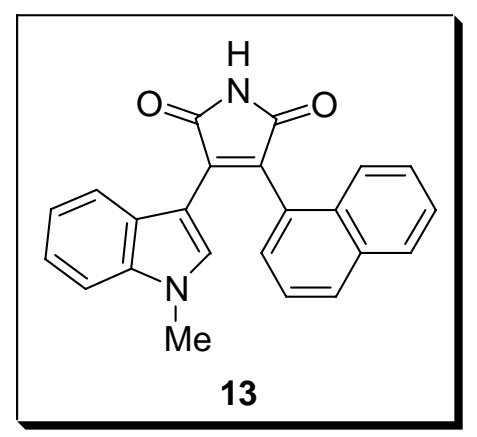

3-(1-Methyl-1H-indol-3-yl)-4-(1-naphthalenyl)-1H-pyrrole-2,5-dione (13): To a mixture of $\mathrm{N}$-methylindole-3-glyoxylamide $(0.21 \mathrm{~g}, 1 \mathrm{mmol})$ and methyl 1 naphthylacetate $(0.5 \mathrm{~mL}, 2.7 \mathrm{mmol})$ in THF $(8 \mathrm{~mL})$ at $0{ }^{\circ} \mathrm{C}$ was slowly added potassium tert-butoxide ( $4 \mathrm{~mL}, 1 \mathrm{M}$ in THF, $4 \mathrm{mmol}$ ). The mixture was stirred for $20 \mathrm{~h}$ allowing the mixture to warm slowly to rt. It was poured into brine-water $(100 \mathrm{~mL}, 1: 1)$ and extracted with ethyl acetate (3 x $50 \mathrm{~mL}$ ). The organic phase was washed with brine (50 mL) and dried $\left(\mathrm{Na}_{2} \mathrm{SO}_{4}\right)$. The solvent was evaporated and the residue was recrystallized from ethyl 
acetate to yield the desired product $13(0.23 \mathrm{~g}, 65 \%)$ as an orange solid: $\mathrm{mp} 257-259{ }^{\circ} \mathrm{C}$; IR (thin film): 1703, 1615, 1515, 1332, 1242, $741 \mathrm{~cm}^{-1} ;{ }^{1} \mathrm{H}-\mathrm{NMR}$ (DMSO-d ${ }_{6}$ ): $\delta 11.18$ (brs, 1H), 8.12 (s, 1H), 8.00 (d, 1H, $J=8.2 \mathrm{~Hz}), 7.95$ (d, 1H, $J=8.2 \mathrm{~Hz}$ ), 7.73 (d, 1H, $J=$ $8.5 \mathrm{~Hz}), 7.52$ (t, $1 \mathrm{H}, J=7.6 \mathrm{~Hz}), 7.43-7.46(\mathrm{~m}, 2 \mathrm{H}), 7.33-7.36(\mathrm{~m}, 2 \mathrm{H}), 6.94(\mathrm{t}, 1 \mathrm{H}, J=$ $7.6 \mathrm{~Hz}), 6.37$ (t, 1H, $J=7.6 \mathrm{~Hz}), 5.96(\mathrm{~d}, 1 \mathrm{H}, J=8.2 \mathrm{~Hz}), 3.83(\mathrm{~s}, 3 \mathrm{H}) ;{ }^{13} \mathrm{C}-\mathrm{NMR}$ (DMSO-d $\mathrm{d}_{6}$ ): $\delta$ 172.6, 172.4, 136.8, 135.1, 135.0, 133.0, 131.3, 129.2, 129.1, 128.7, 128.3, 128.0, 126.3, 126.1, 125.7, 125.3, 125.1, 122.0, 120.4, 120.0, 110.4, 104.0, 33.0; LRMS (EI): $m / z 352\left(\mathrm{M}^{+}\right), 200,158,141$ (100\%), 115; HRMS (EI): calcd for $\mathrm{C}_{23} \mathrm{H}_{16} \mathrm{~N}_{2} \mathrm{O}_{2}$ : 352.1212, found: 352.1213.

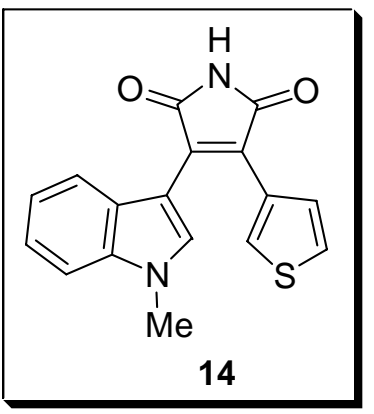

3-(1-Methyl-1H-indol-3-yl)-4-(3-thienyl)-1H-pyrrole-2,5-dione (14): To a mixture of $\mathrm{N}$-methylindole-3-glyoxylamide $(0.21 \mathrm{~g}, 1 \mathrm{mmol})$ and methyl thiophene-3-acetate $(0.43$ g, $2.7 \mathrm{mmol})$ in THF $(8 \mathrm{~mL})$ at $0{ }^{\circ} \mathrm{C}$ was slowly added potassium tert-butoxide $(4 \mathrm{~mL}, 1$ $\mathrm{M}$ in THF, $4 \mathrm{mmol}$ ). The mixture was stirred for $14 \mathrm{~h}$ allowing the mixture to warm slowly to rt. It was poured into brine-water $(100 \mathrm{~mL}, 1: 1)$ and extracted with ethyl acetate (3 x $50 \mathrm{~mL})$. The organic phase was washed with brine $(50 \mathrm{~mL})$ and dried $\left(\mathrm{Na}_{2} \mathrm{SO}_{4}\right)$. The solvent was evaporated and the residue was recrystallized from ethyl acetate to yield the desired product 14 (0.26 g, 84\%) as a yellowish-orange solid: mp 223-225 ${ }^{\circ} \mathrm{C}$; IR (thin film): 1758, 1702, 1619, 1526, 1333, $742 \mathrm{~cm}^{-1}$; ${ }^{1} \mathrm{H}-\mathrm{NMR}$ (DMSO-d 6 ): $\delta 11.06$ (s, 1H), 7.94 (s, 1H), 7.81-7.82 (m, 1H), 7.51 (d, 1H, $J=8.2 \mathrm{~Hz}$ ), 7.46-7.48 (m, 1H), 7.17 (t, 1H, $J=7.6 \mathrm{~Hz}$ ), 7.05 (dd, 1H, $J=5.0 \mathrm{~Hz}, 1.1 \mathrm{~Hz}), 6.86$ (dt, 1H, $J=7.5 \mathrm{~Hz}, 0.9 \mathrm{~Hz}), 6.58$ (d, $1 \mathrm{H}, J=7.9 \mathrm{~Hz}$ ), 3.91 (s, 3H); ${ }^{13} \mathrm{C}-\mathrm{NMR}\left(\mathrm{DMSO}-\mathrm{d}_{6}\right): \delta 172.4,172.3,137.1,134.2,130.5$, 130.4, 128.2, 128.0, 125.6, 125.2, 124.7, 122.1, 121.2, 120.1, 110.6, 103.2, 33.0; LRMS (EI): $m / z 308\left(\mathrm{M}^{+}, 100 \%\right), 275,237,156,118,97$; HRMS (EI): calcd for $\mathrm{C}_{17} \mathrm{H}_{12} \mathrm{~N}_{2} \mathrm{O}_{2} \mathrm{~S}$ : 308.0620, found: 308.0618. 


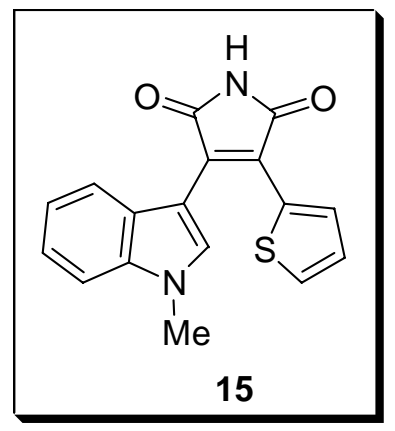

3-(1-Methyl-1H-indol-3-yl)-4-(2-thienyl)-1H-pyrrole-2,5-dione (15): To a mixture of $\mathrm{N}$-methylindole-3-glyoxylamide $(0.21 \mathrm{~g}, 1 \mathrm{mmol})$ and methyl thiophene-2-acetate $(0.53$ g, $3.3 \mathrm{mmol})$ in THF $(8 \mathrm{~mL})$ at $0{ }^{\circ} \mathrm{C}$ was slowly added potassium tert-butoxide $(6 \mathrm{~mL}, 1$ $\mathrm{M}$ in THF, $6 \mathrm{mmol}$ ). The mixture was stirred for $17 \mathrm{~h}$ allowing the mixture slowly to warm to rt. It was poured into brine-water $(100 \mathrm{~mL}, 1: 1)$ and extracted with ethyl acetate (3 x $50 \mathrm{~mL})$. The organic phase was washed with brine $(50 \mathrm{~mL})$ and dried $\left(\mathrm{Na}_{2} \mathrm{SO}_{4}\right)$. The solvent was evaporated and the residue was recrystallized from ethyl acetate to yield the desired product 15 (0.23 g, 75\%) as a bright red solid: $\mathrm{mp} 243-245{ }^{\circ} \mathrm{C}$; IR (thin film): 1756, 1702, 1594, 1522, 1328, $741 \mathrm{~cm}^{-1}$; ${ }^{1} \mathrm{H}-\mathrm{NMR}$ (DMSO-d $\mathrm{d}_{6}$ ): $\delta 11.17$ (s, 1H), 7.92 (s, 1H), 7.72 (d, 1H, $J=5.2 \mathrm{~Hz}$ ), 7.55 (d, 1H, $J=7.9 \mathrm{~Hz}$ ), 7.18-7.21 (m, 2H), 7.02-7.04 (m, 1H), 6.95 (t, 1H, $J=7.9 \mathrm{~Hz}$ ), 6.72 (d, $1 \mathrm{H}, J=8.2 \mathrm{~Hz}$ ), 3.92 (s, 3H); ${ }^{13} \mathrm{C}-\mathrm{NMR}$ (DMSO$\left.\mathrm{d}_{6}\right): \delta 172.1,172.0,137.1,134.2,130.5,130.4,128.6,127.0,124.6,124.2,122.1,121.6$, 120.1, 110.8, 102.8, 33.0; LRMS (EI): $\mathrm{m} / \mathrm{z} 308$ (M+1 100\%), 275, 237, 222, 156, 118, 97; HRMS (EI): calcd for $\mathrm{C}_{17} \mathrm{H}_{12} \mathrm{~N}_{2} \mathrm{O}_{2} \mathrm{~S}$ : 308.0620, found: 308.0625.

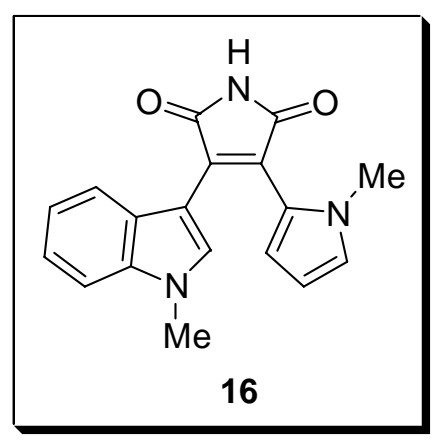

1-Methyl-4'-(1-methyl-1H-indol-3-yl)-[2,3'-bi-1H-pyrrole]-2',5'-dione (16): To a mixture of $N$-methylindole-3-glyoxylamide $(0.21 \mathrm{~g}, 1 \mathrm{mmol})$ and methyl 1 methylpyrrole-2-acetate $(0.4 \mathrm{~mL}, 2.7 \mathrm{mmol})$ in THF $(8 \mathrm{~mL})$ at $0{ }^{\circ} \mathrm{C}$ was slowly added potassium tert-butoxide ( $4 \mathrm{~mL}, 1 \mathrm{M}$ in THF, $4 \mathrm{mmol}$ ). The mixture was stirred for $15 \mathrm{~h}$ allowing the mixture to warm slowly to rt. It was poured into brine-water $(100 \mathrm{~mL}, 1: 1)$ and extracted with ethyl acetate $(3 \times 50 \mathrm{~mL})$. The organic phase was washed with brine $(50 \mathrm{~mL})$ and dried $\left(\mathrm{Na}_{2} \mathrm{SO}_{4}\right)$. The solvent was evaporated and the residue was recrystallized from ethyl acetate to yield the desired product $16(0.23 \mathrm{~g}, 75 \%)$ as a bright red solid: mp 240-242 ${ }^{\circ} \mathrm{C}$; IR (thin film): 1758, 1705, 1621, 1535, 1332, $739 \mathrm{~cm}^{-1}$; ${ }^{1} \mathrm{H}-$ NMR (DMSO-d D $_{6}: \delta 10.99$ (s, 1H), 8.08 (s, 1H), 7.46 (d, 1H, $\left.J=8.2 \mathrm{~Hz}\right), 7.13$ (t, 1H, $J=$ 
$7.6 \mathrm{~Hz}), 6.84$ (s, 1H), 6.76 (t, 1H, $J=7.5 \mathrm{~Hz}), 6.32$ (d, 1H, $J=8.2 \mathrm{~Hz}), 6.27-6.28$ (m, $1 \mathrm{H}), 6.14-6.16(\mathrm{~m}, 1 \mathrm{H}), 3.88(\mathrm{~s}, 3 \mathrm{H}), 3.13(\mathrm{~s}, 3 \mathrm{H}) ;{ }^{13} \mathrm{C}-\mathrm{NMR}\left(\mathrm{DMSO}-\mathrm{d}_{6}\right): \delta 172.3,172.2$, 136.9, 134.7, 131.3, 125.6, 125.3, 123.1, 122.3, 121.7, 120.7, 120.3, 113.4, 110.3, 108.7, 104.6, 34.7, 33.0; LRMS (EI): m/z $305\left(\mathrm{M}^{+}\right)$, 274, 242, 167, 138, 121, 108, 94 (100\%); HRMS (EI): calcd for $\mathrm{C}_{18} \mathrm{H}_{15} \mathrm{~N}_{3} \mathrm{O}_{2}$ : 305.1164, found: 305.1166 .

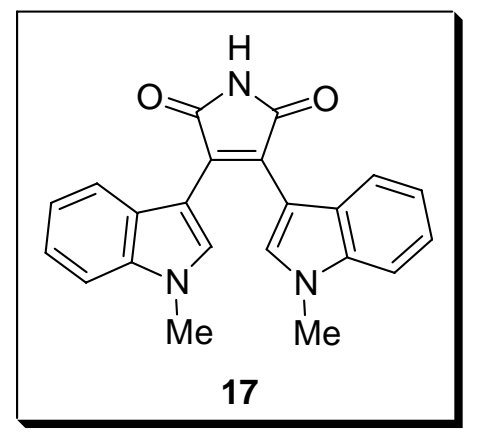

3,4-Bis(1-methyl-1H-indol-3-yl)-1H-pyrrole-2,5-dione (17): To a mixture of $\mathrm{N}$ methylindole-3-glyoxylamide $(0.21 \mathrm{~g}, 1 \mathrm{mmol})$ and 1-methyl indole-3-acetate (0.55 g, $2.7 \mathrm{mmol})$ in THF $(8 \mathrm{~mL})$ at $0{ }^{\circ} \mathrm{C}$ was slowly added potassium tert-butoxide $(4 \mathrm{~mL}, 1 \mathrm{M}$ in THF, $4 \mathrm{mmol}$ ). The mixture was stirred for $16 \mathrm{~h}$ allowing the mixture to slowly warm to rt. It was poured into brine-water $(100 \mathrm{~mL}, 1: 1)$ and extracted with ethyl acetate $(2 \mathrm{x}$ $50 \mathrm{~mL})$. The organic phase was washed with brine $(50 \mathrm{~mL})$ and dried $\left(\mathrm{Na}_{2} \mathrm{SO}_{4}\right)$. The solvent was evaporated and the residue was recrystallized from ethyl acetate to yield the desired product $17(0.22 \mathrm{~g}, 62 \%)$ as a red solid: mp 357-359 ${ }^{\circ} \mathrm{C} ;{ }^{1} \mathrm{H}-\mathrm{NMR}\left(\mathrm{DMSO}-\mathrm{d}_{6}\right): \delta$ 10.93 (s, 1H), 7.80 (s, 2H), 7.42 (d, 2H, $J=8.2 \mathrm{~Hz}$ ), 7.03 (t, 2H, $J=7.6 \mathrm{~Hz}$ ), 6.75 ( d, $2 \mathrm{H}, J=8.2 \mathrm{~Hz}$ ), 6.65 (t, 2H, $J=7.5 \mathrm{~Hz}$ ), $3.84(\mathrm{~s}, 6 \mathrm{H}) ;{ }^{13} \mathrm{C}-\mathrm{NMR}\left(\mathrm{DMSO}-\mathrm{d}_{6}\right): \delta$ 173.0, 136.5, 133.0, 127.0, 125.9, 121.7, 121.1, 119.5, 110.1, 104.7, 32.9; LRMS (ESI+): $\mathrm{m} / \mathrm{z}$ $378[\mathrm{M}+\mathrm{Na}]^{+}, 356[\mathrm{M}+\mathrm{H}]^{+}$; HRMS (ESI+): calcd for $\mathrm{C}_{22} \mathrm{H}_{18} \mathrm{~N}_{3} \mathrm{O}_{2}: 356.1399[\mathrm{M}+\mathrm{H}]$, found: 356.1405.

\section{References and notes:}

1. Roy, S.; Eastman, A.; Gribble, G. W. Tetrahedron 2006, 62, 7838-7845.

2. Heinzelman, S. Prog. Drug Res. 1963, 6, 75-150.

3. Roy, S.; Eastman, A.; Gribble, G. W. Org. Biomol. Chem. 2006, 4, 3228-3234.

4. Rahman, A.; Sultana; M.; Hassan, I. Tetrahedron Lett.1983, 24, 1845-1848. 


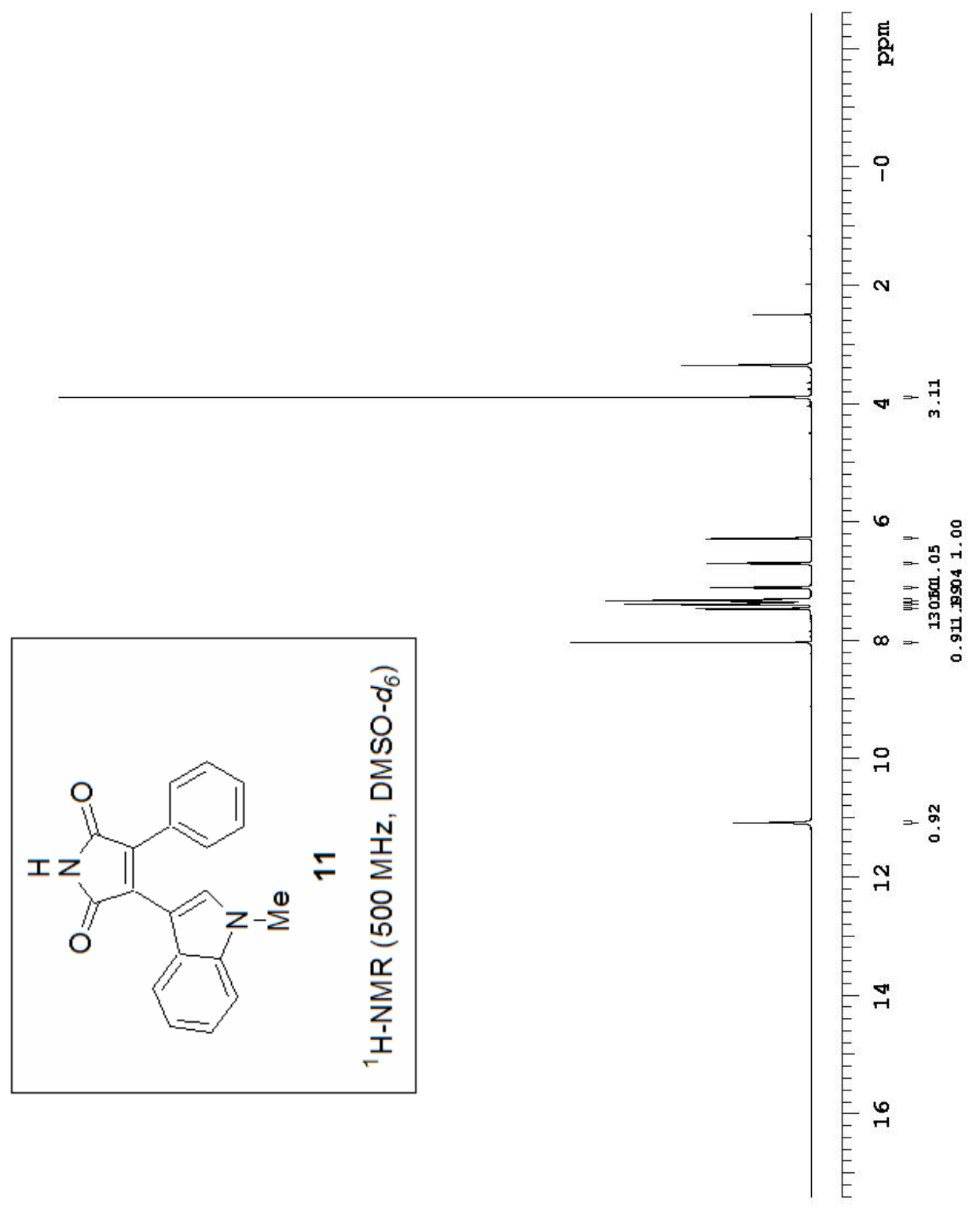




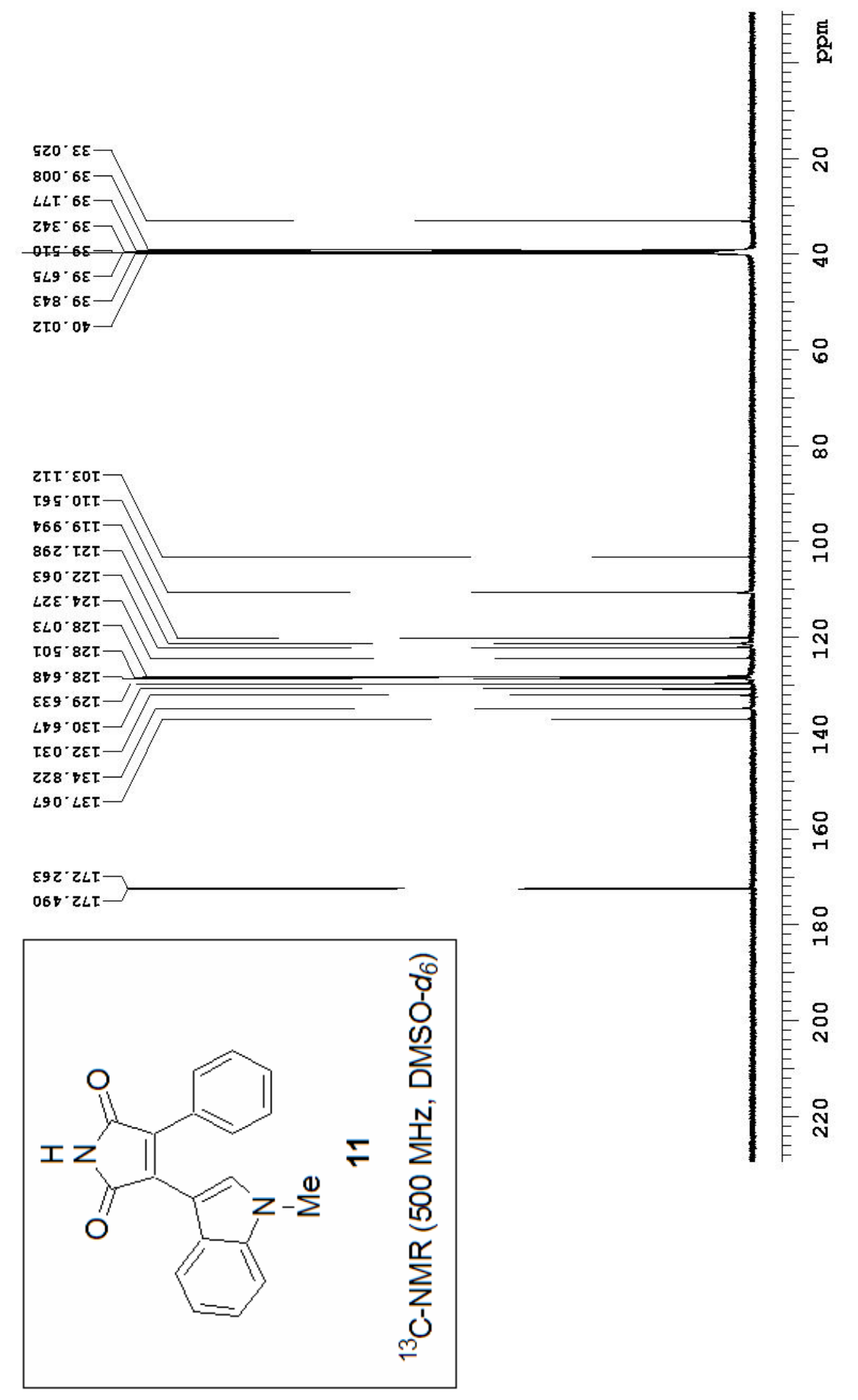




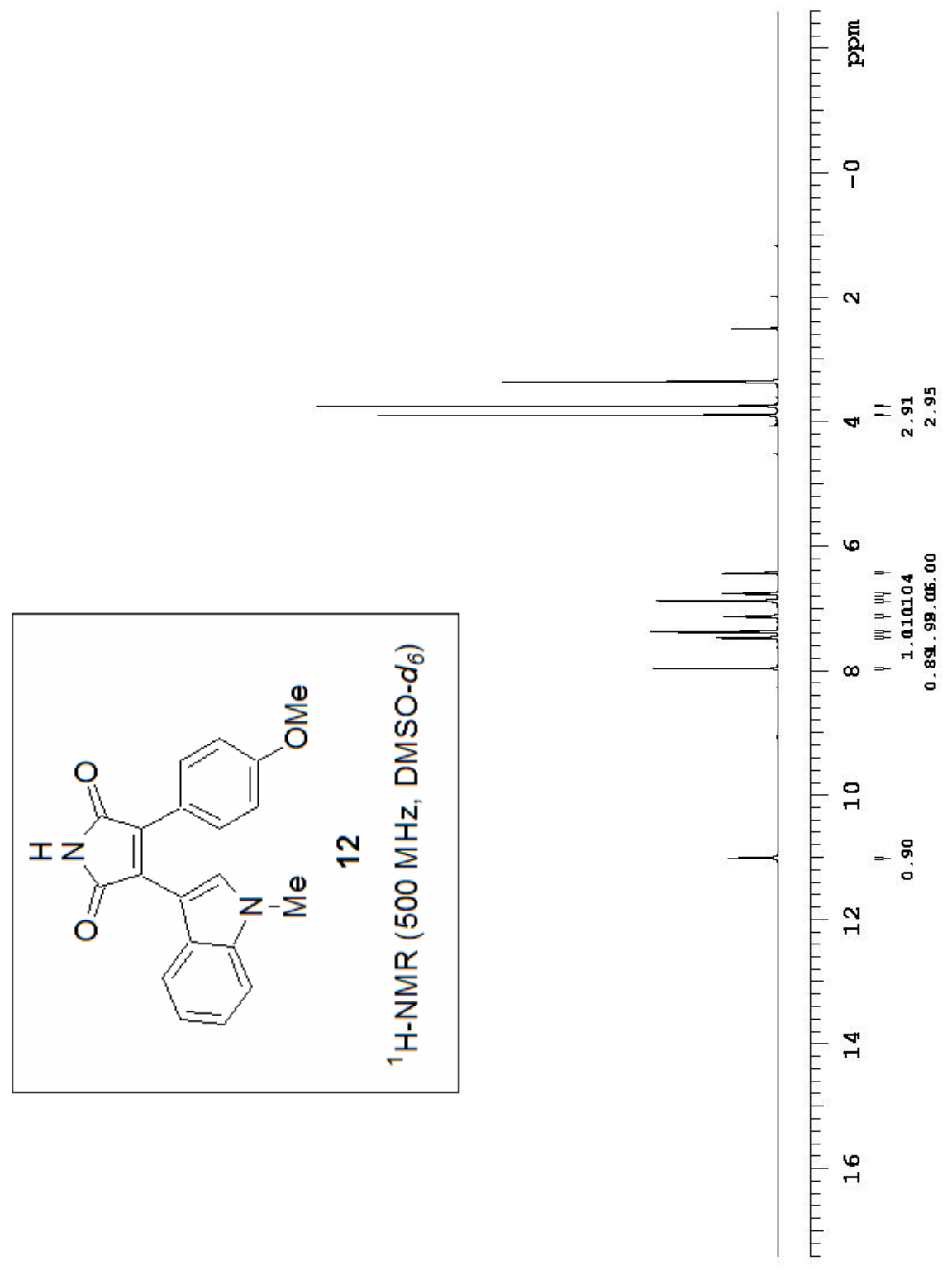




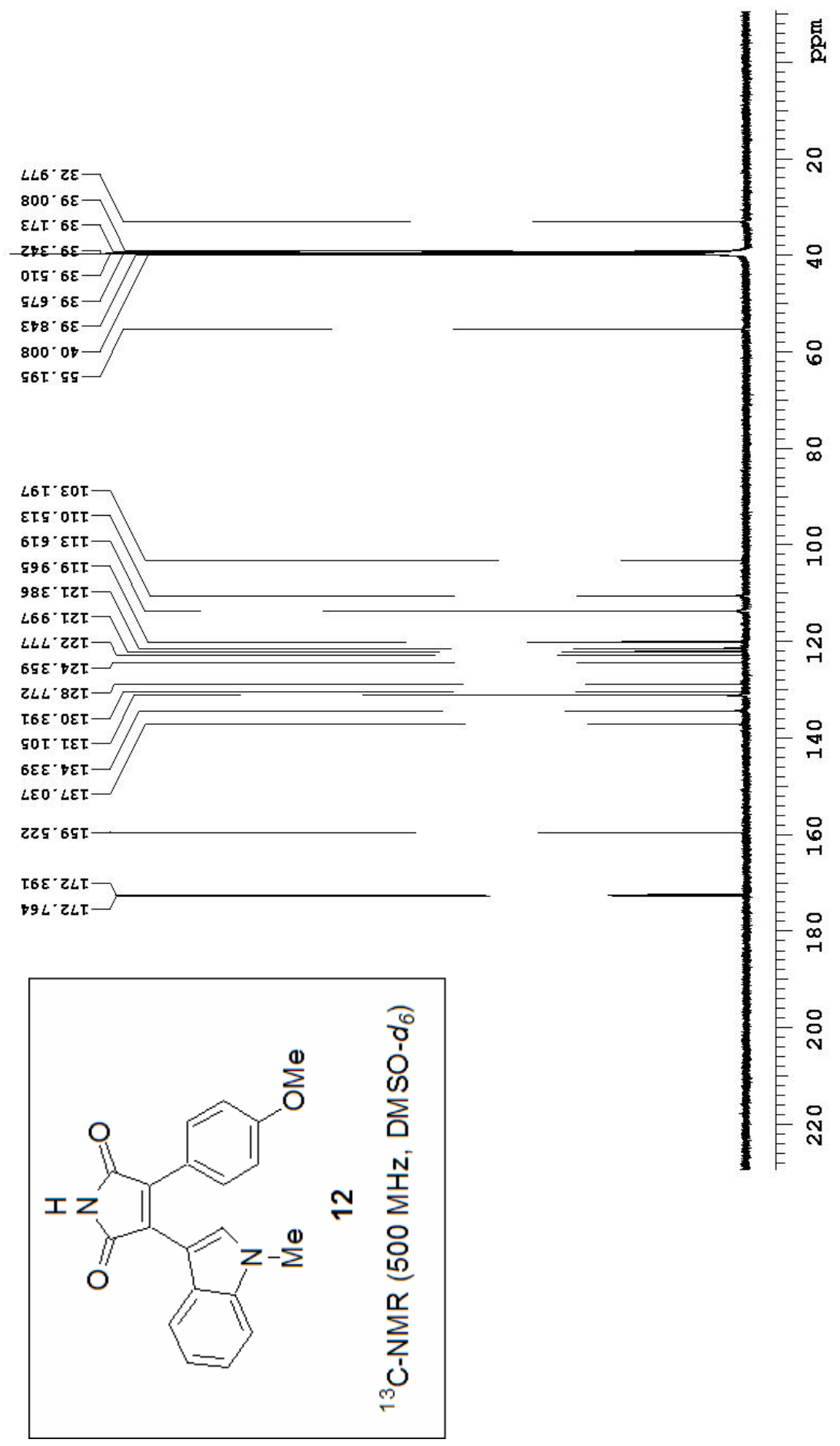




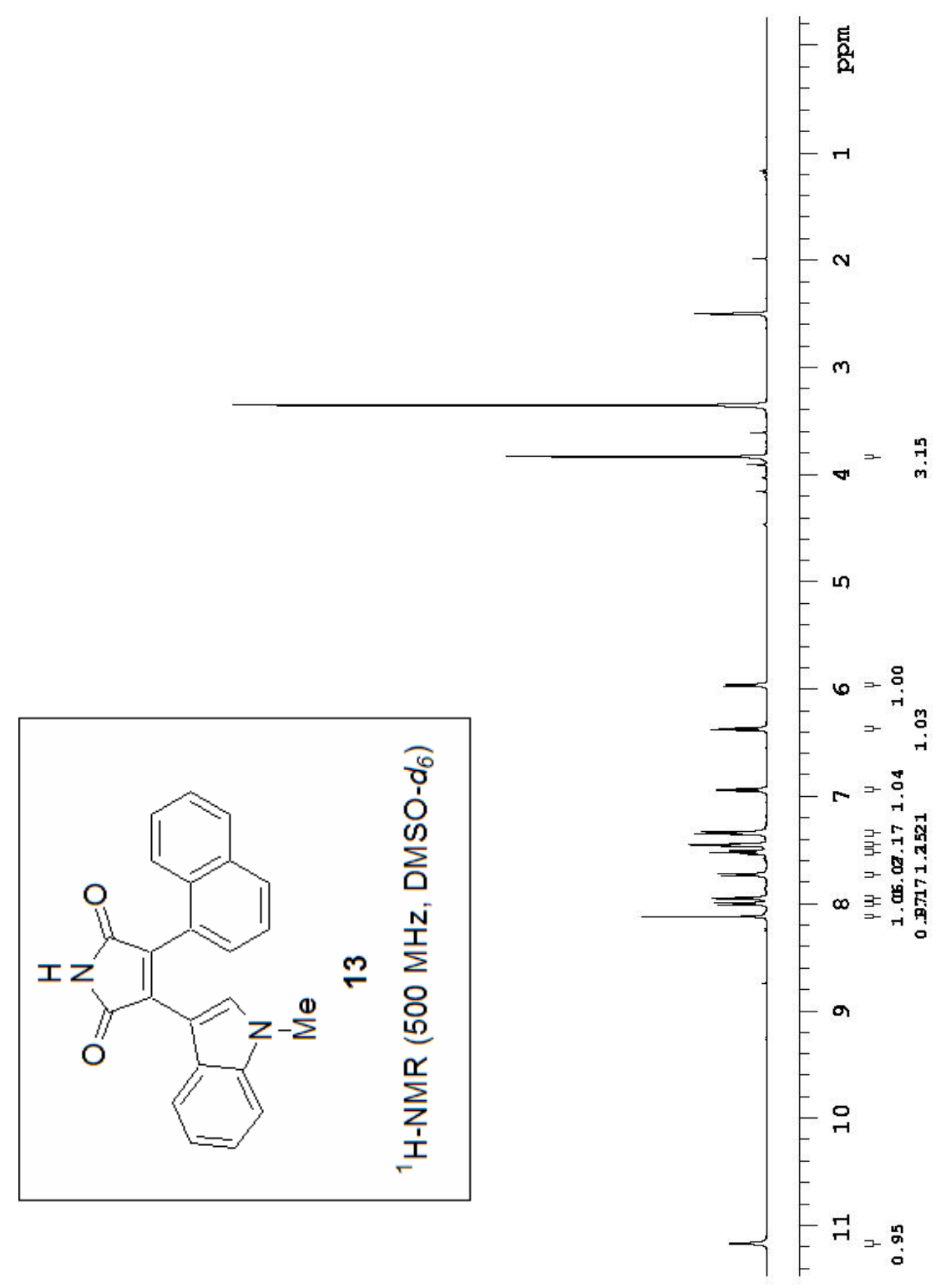




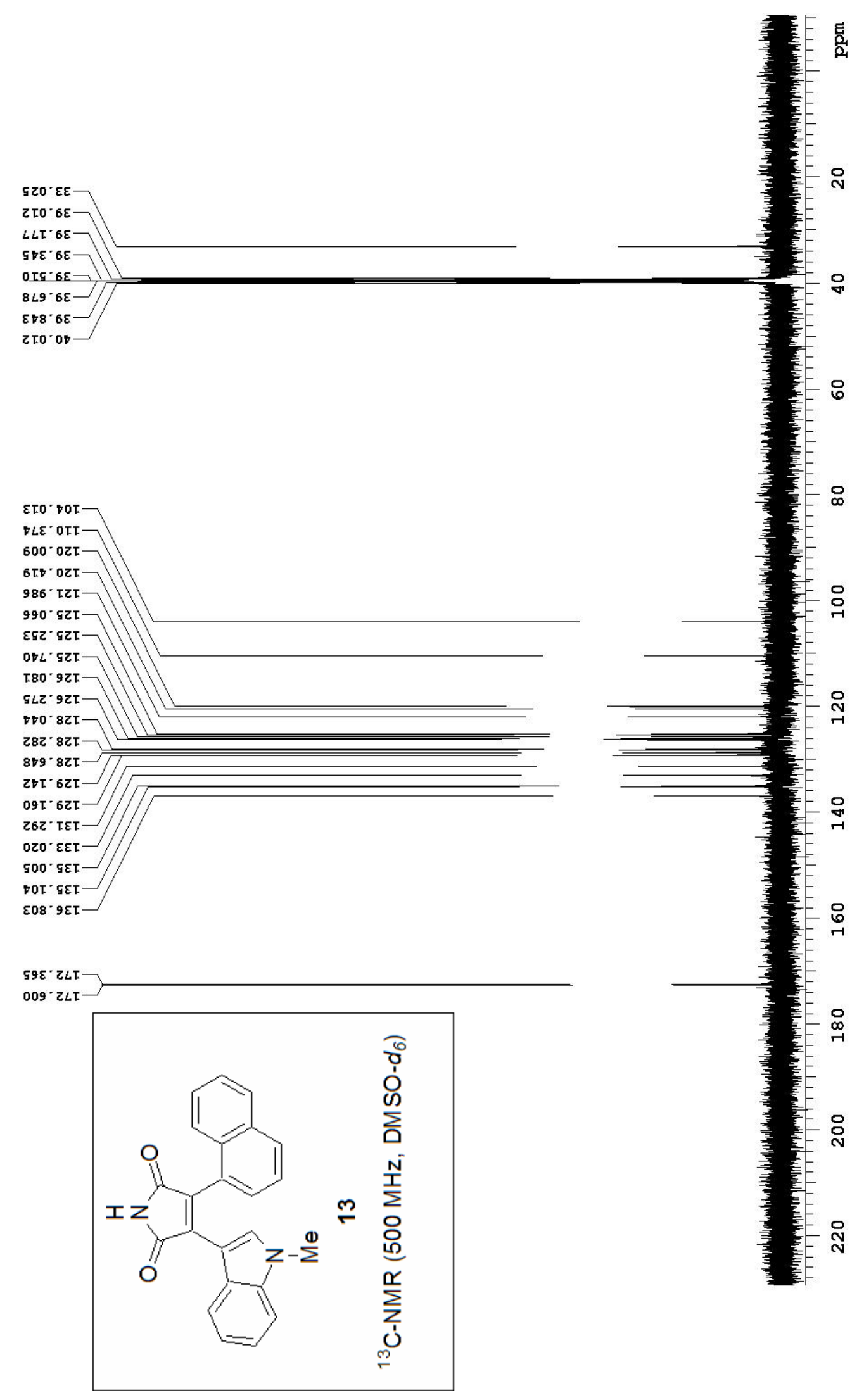




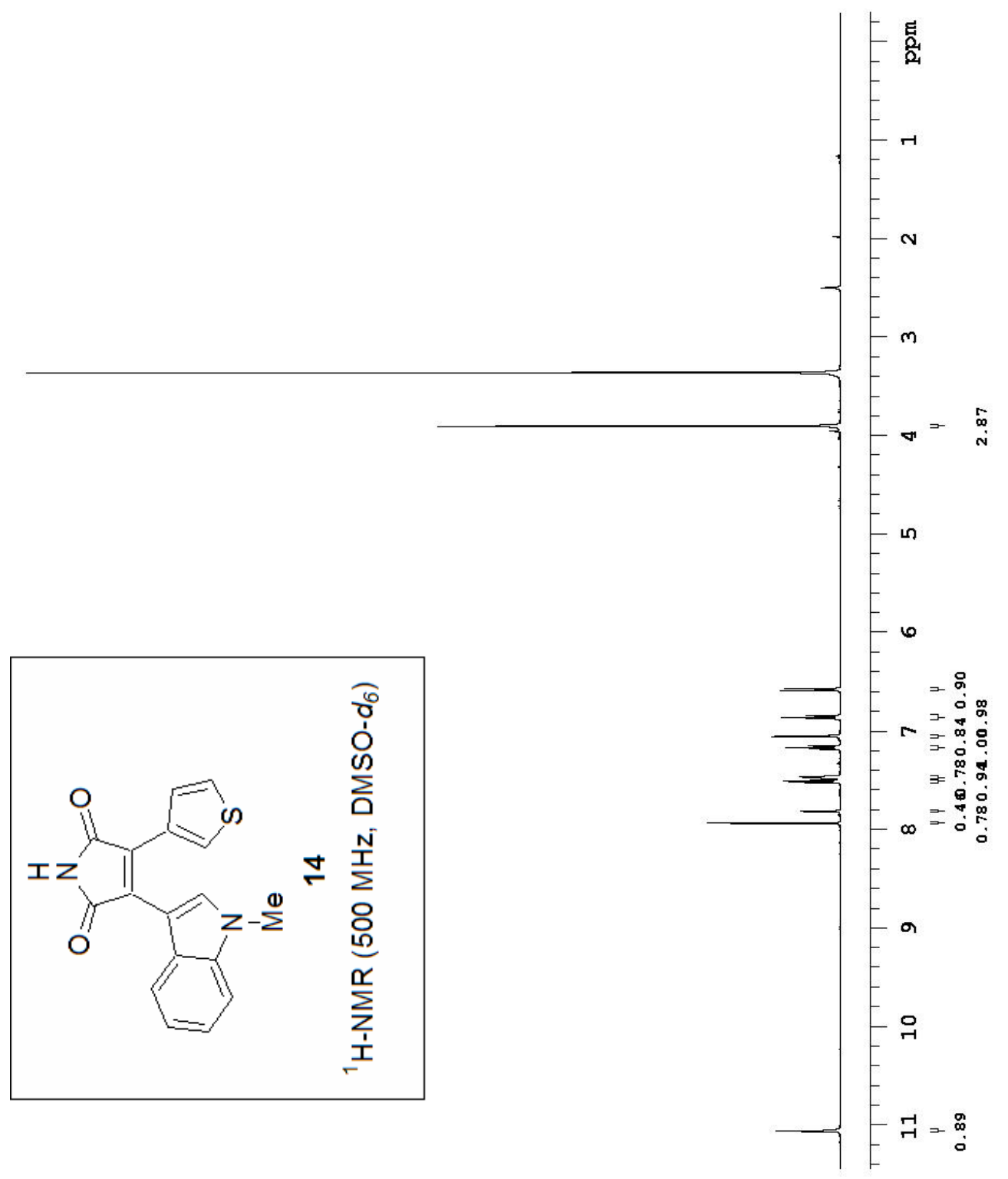




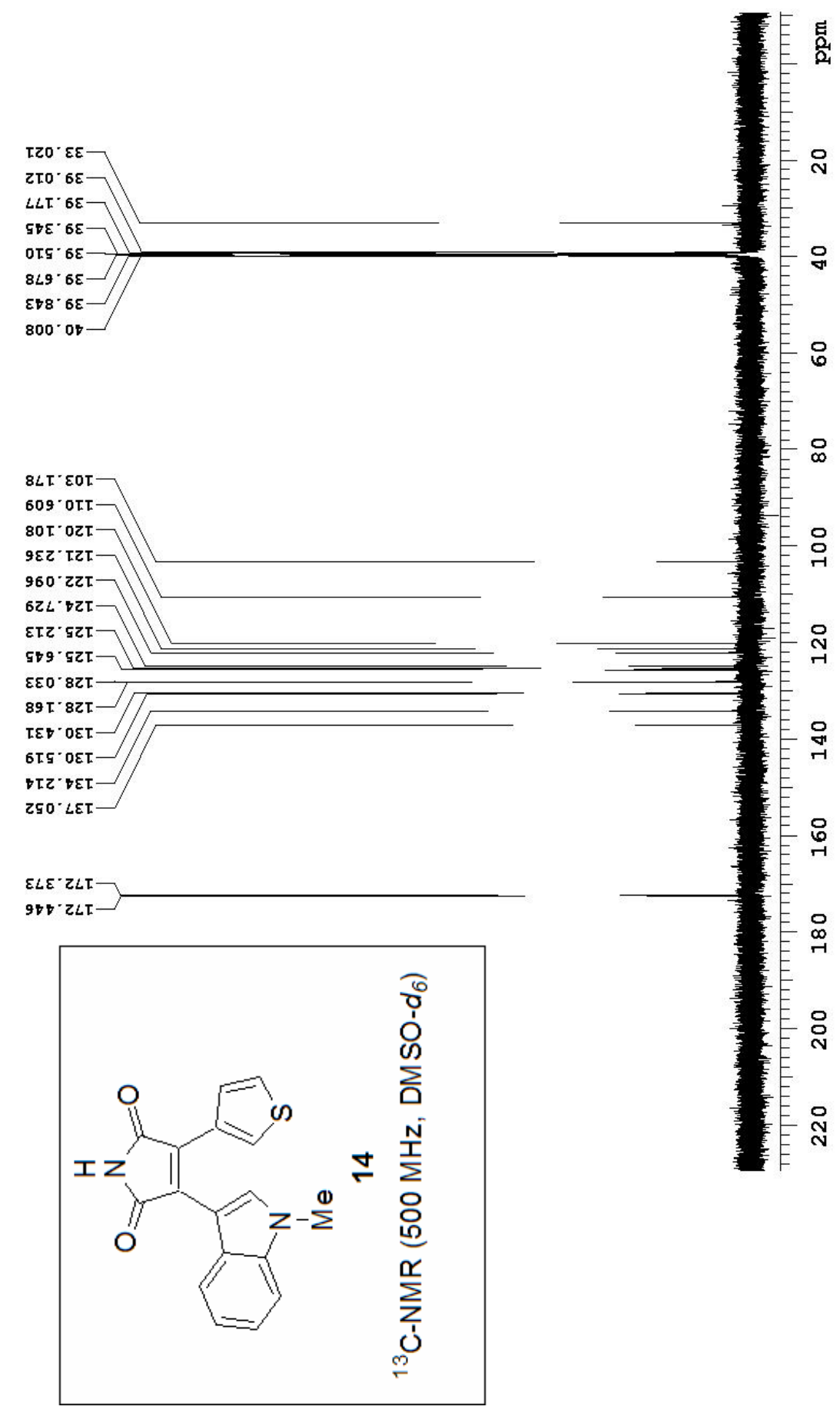




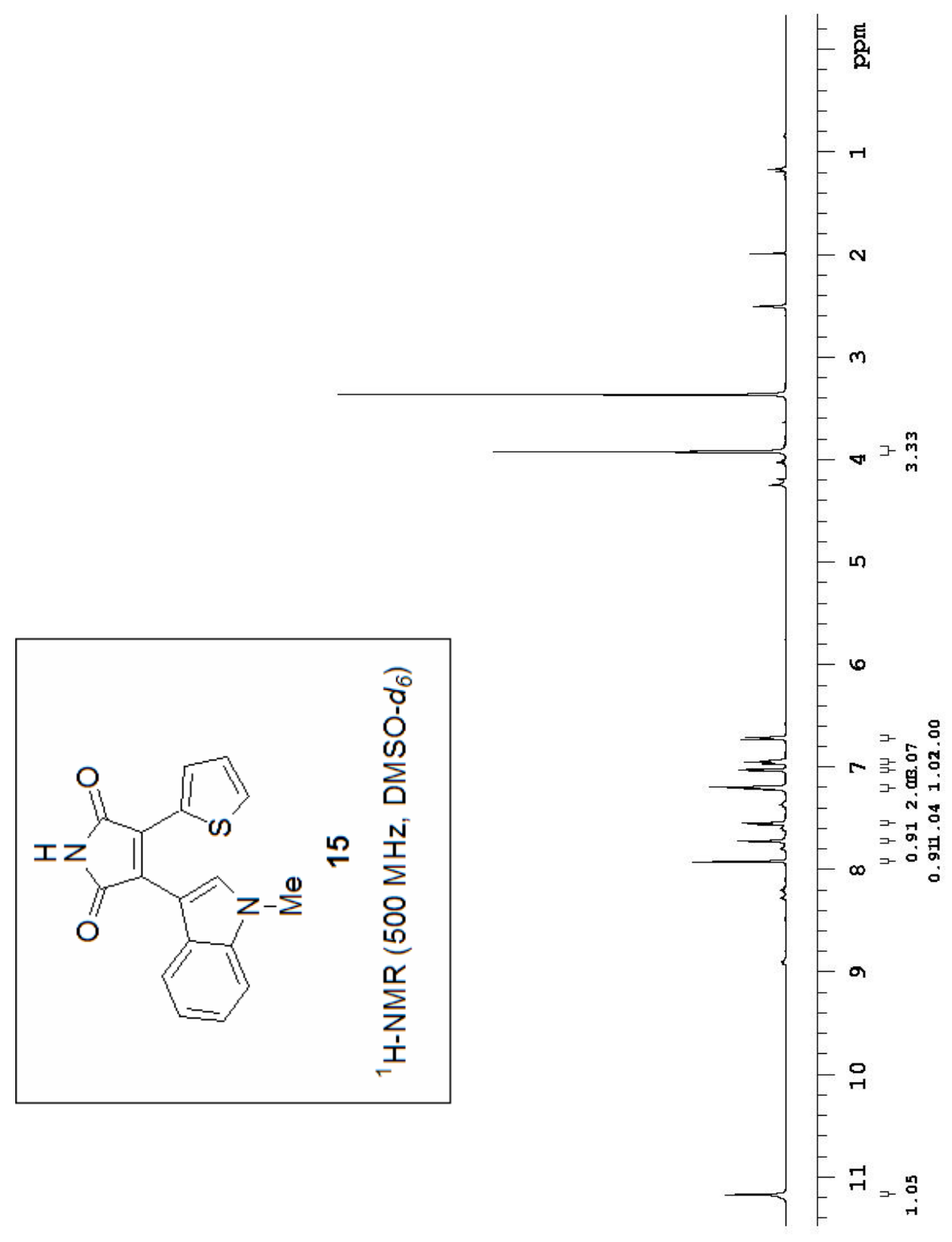




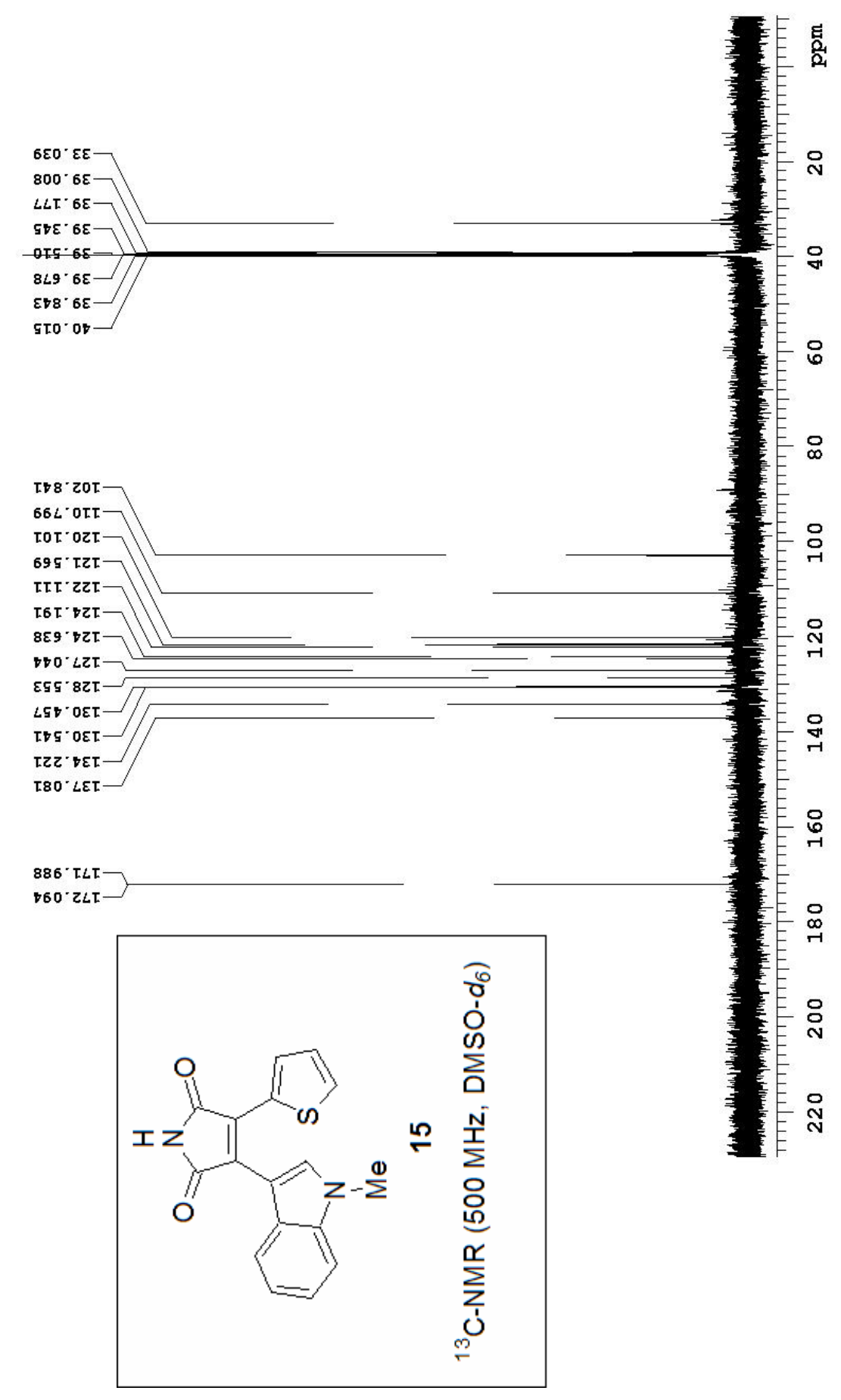




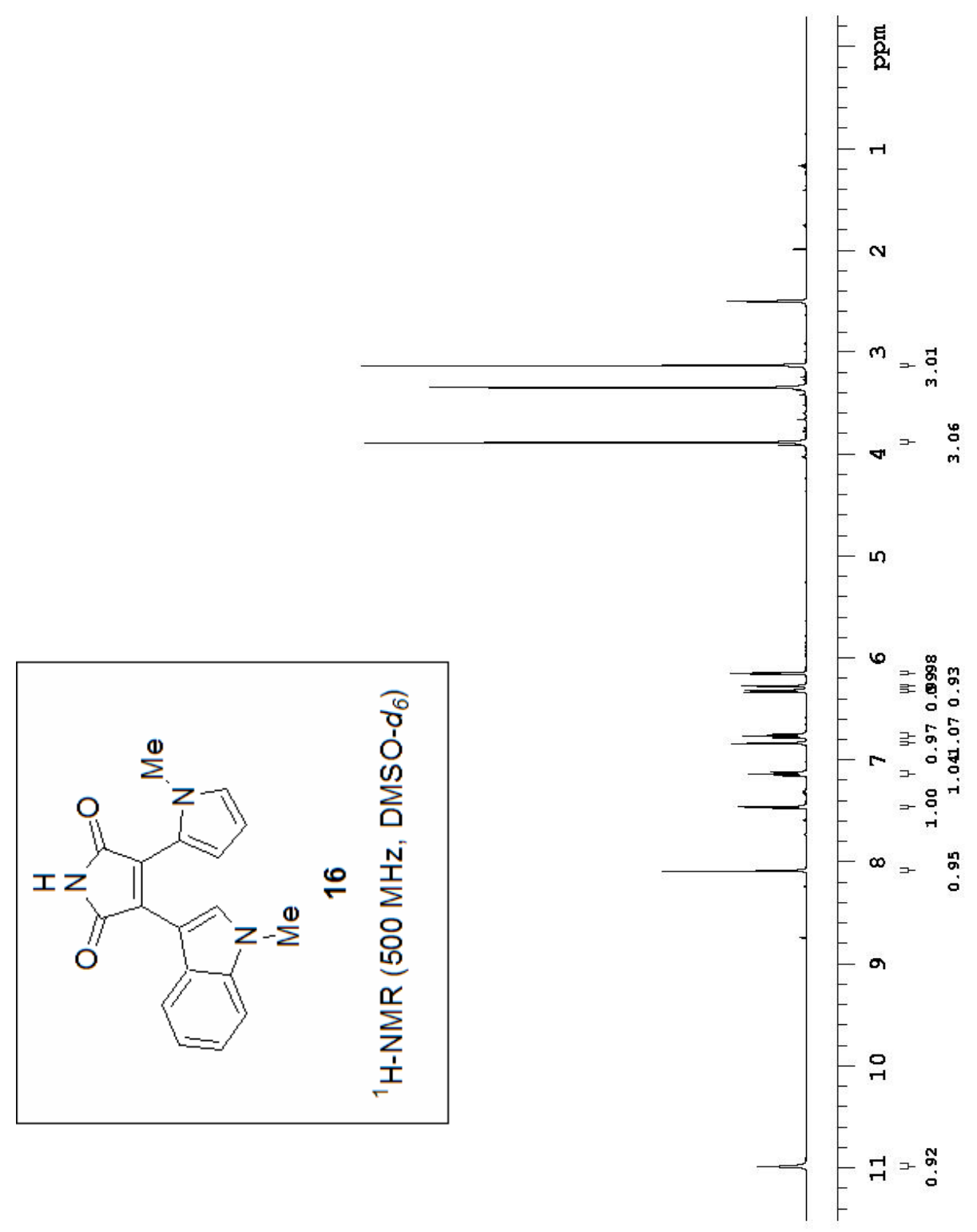




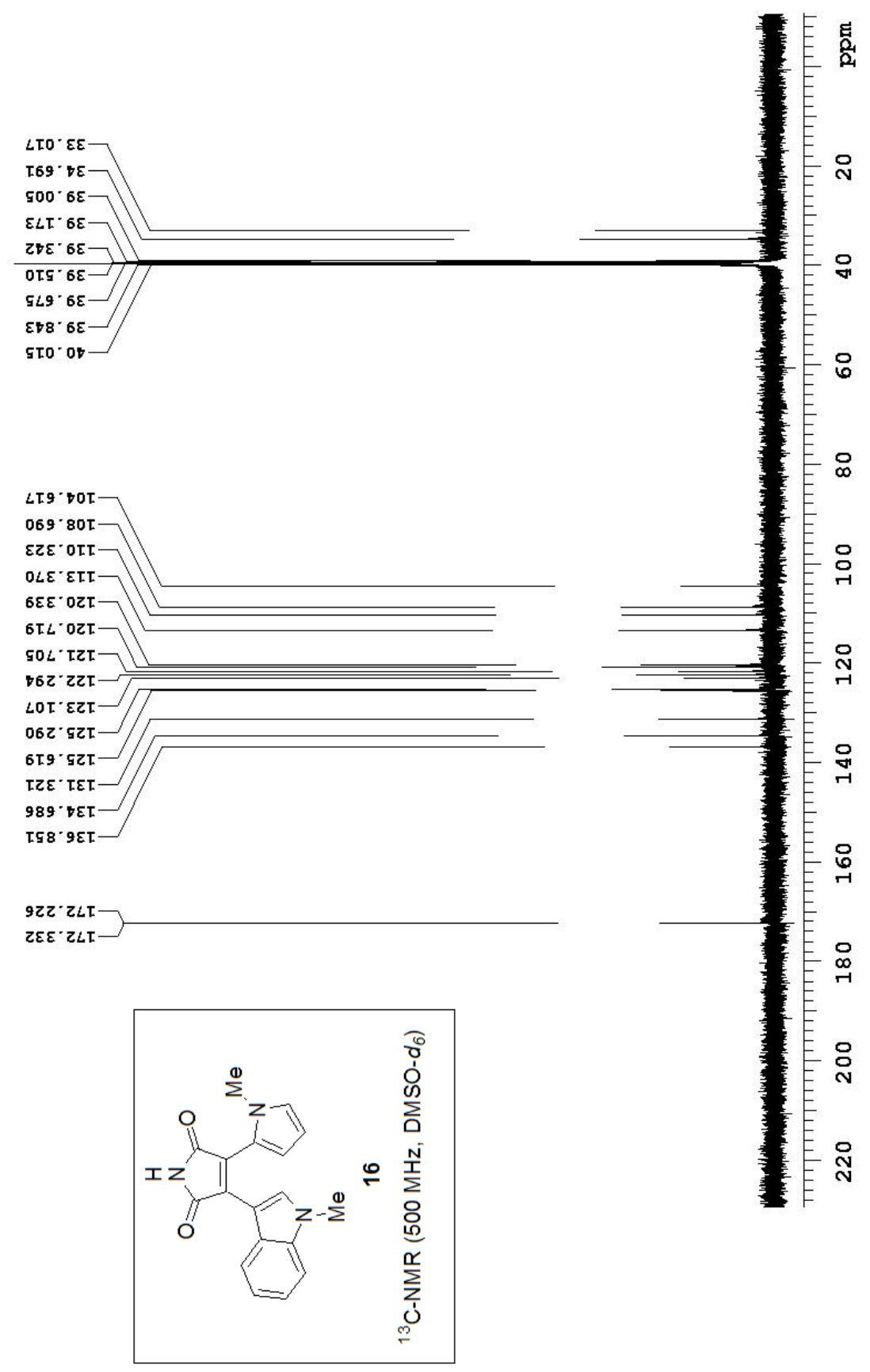




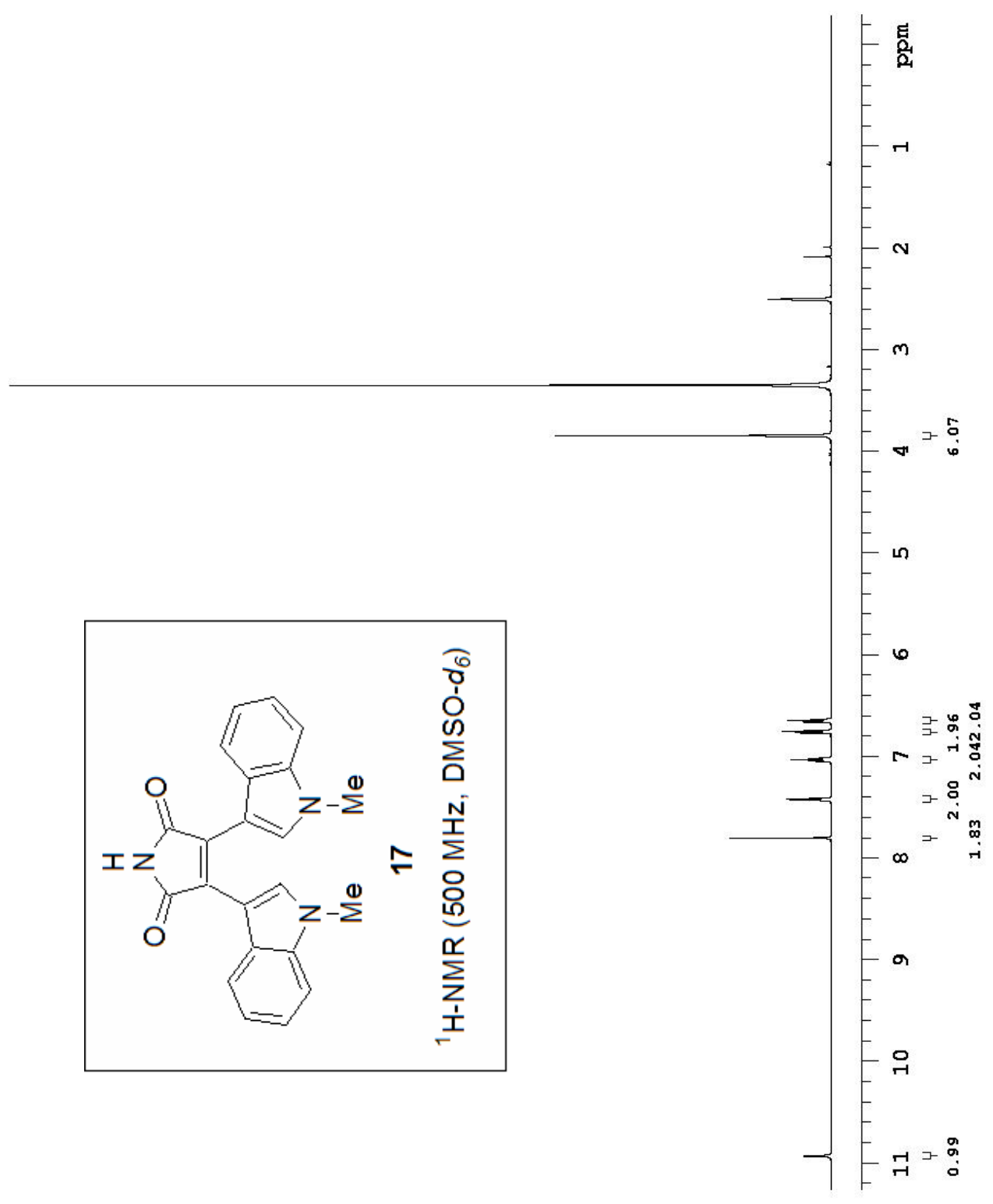




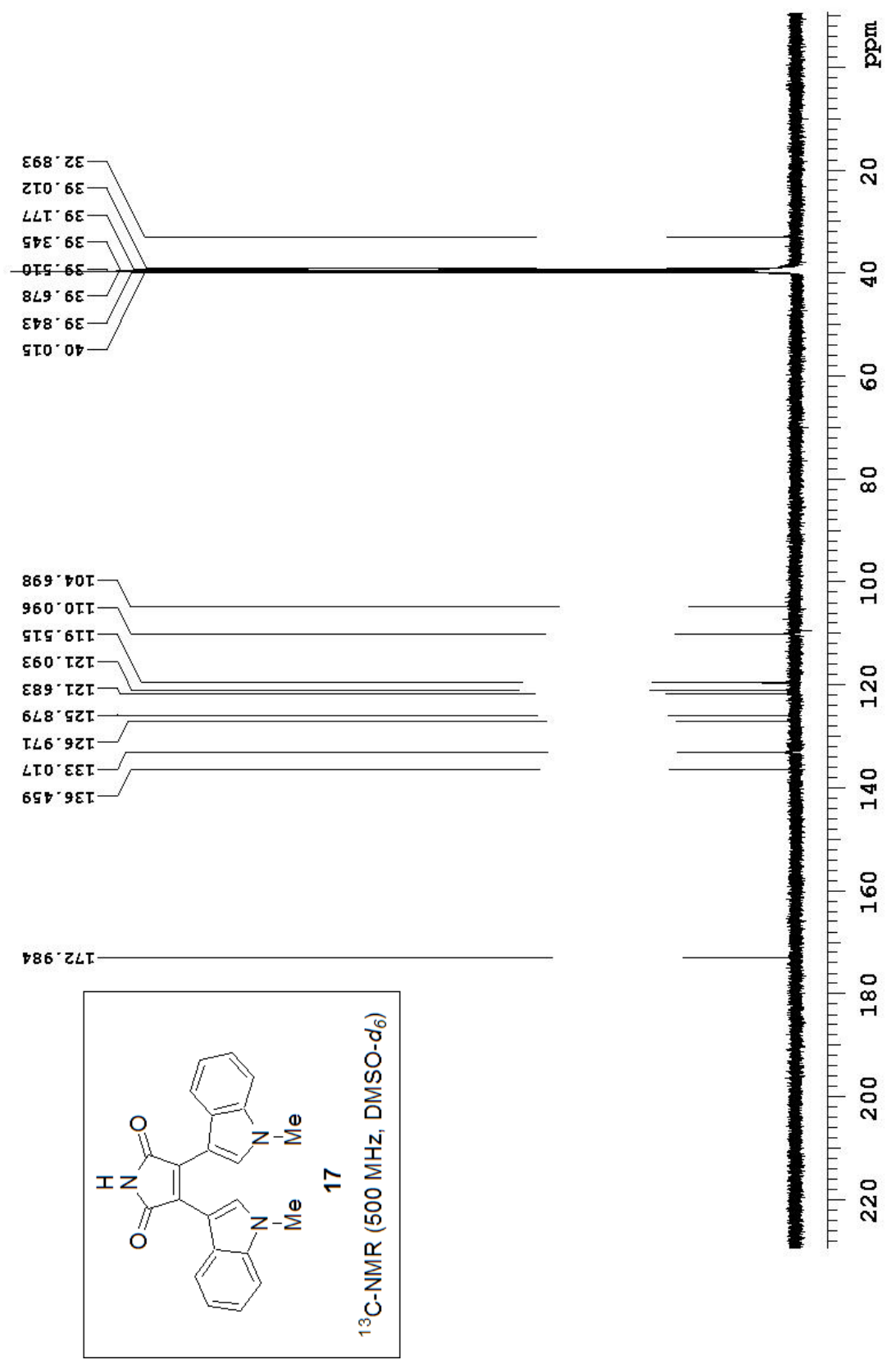

